\title{
Comparison of Three Molecular Simulation Approaches for Cyclodextrin-Ibuprofen Complexation
}

\author{
Runmiao Wang, ${ }^{1}$ Hui Zhou, ${ }^{1}$ Shirley W. I. Siu, ${ }^{2}$ Yong Gan, ${ }^{3}$ \\ Yitao Wang, ${ }^{1}$ and Defang Ouyang ${ }^{1}$ \\ ${ }^{1}$ State Key Laboratory of Quality Research in Chinese Medicine, Institute of Chinese Medical Sciences, University of Macau, Macau \\ ${ }^{2}$ Faculty of Science and Technology, University of Macau, Macau \\ ${ }^{3}$ Shanghai Institute of Materia Medica, Chinese Academy of Sciences, Shanghai 201203, China \\ Correspondence should be addressed to Defang Ouyang; defangouyang@umac.mo
}

Received 7 May 2015; Accepted 21 May 2015

Academic Editor: Xingfu Xu

Copyright (C) 2015 Runmiao Wang et al. This is an open access article distributed under the Creative Commons Attribution License, which permits unrestricted use, distribution, and reproduction in any medium, provided the original work is properly cited.

\begin{abstract}
Cyclodextrins are widely used for the solubilisation of poorly soluble drugs in the formulations. However, current cyclodextrin formulation development strongly depends on trial-and-error in the laboratory, which is time-consuming and high cost. The aim of this research was to compare three modeling approaches (Docking, molecular dynamics (MD), and quantum mechanics $(\mathrm{QM})$ ) for cyclodextrin/drug complexation. Ibuprofen was used as a model drug. Binding free energy from three simulation methods was calculated as an important parameter to compare with the experimental results. Docking results from AutoDock Vina program showed that the scoring of complexation capability between ibuprofen and cyclodextrins is alpha $(\alpha)$, gamma $(\gamma)$, beta $(\beta)$, and HP-beta-cyclodextrins, which indicated similar ranking with the results from phase, solubility diagram experiments. MD simulation indicated that ibuprofen could form the stable complexes with $\beta$-, $\gamma$-, and HP- $\beta$-cyclodextrins, but not for alpha cyclodextrin. Binding free energies from the MD simulation for $\beta-, \gamma-$, and HP- $\beta$-cyclodextrins were $-3.67,-0.67$, and $-3.87 \mathrm{kcal} / \mathrm{mol}$, individually. The enthalpies of QM simulation for $\beta-, \gamma-$, and HP- $\beta$-cyclodextrins were $-17.22,-14.75$, and $-20.28 \mathrm{kcal} / \mathrm{mol}$, respectively. Results from all three modeling approaches showed similar ranking between ibuprofen and four cyclodextrin molecules as the experimental data. However, MD simulation with entropy calculation had the closest value to experimental data for $\beta$ and HP-beta-cyclodextrins. Thus, MD simulation with MM-PBSA method may be fit to in silico screen for cyclodextrin formulations.
\end{abstract}

\section{Introduction}

Cyclodextrins (CDs) are natural pharmaceutical ingredients for the solubility-enhancement of poorly soluble drugs for over half century. Chemical composition of cyclodextrin consists of D-glucopyranoses, which are linked by alpha 1,4-glycosidic bonds to form the macrocyclic ring conformations. Cyclodextrins are classified into alpha, beta, and gamma cyclodextrins, comprised of six, seven, and eight Dglucopyranose residues, respectively [1]. These macrocyclic structures have hydrophilic outer with hydroxyl groups, while the nonpolar inner cavity is hydrophobic [1]. The unique structure enables CDs to accommodate poorly watersoluble drugs to form drug-inclusion complexes. However, cyclodextrins are poor across most of the biological membranes due to their high molecular weight and inherent hydrophilicity [2]. There are over 35 commercially available pharmaceutical formulations including various natural or chemically modified cyclodextrins [1]. Some experimental techniques are able to characterize drug-cyclodextrins inclusion complexes, such as thermoanalytical [3], spectroscopic [4], microscopic [5], and chromatographic techniques [6]. However, current cyclodextrin formulation development mainly depends on trial-and-error by formulation scientists in the laboratory, which is time-consuming and high cost.

Molecule modeling method is able to investigate the structure, dynamics, and energetics of cyclodextrin systems. From the search of Web of Science, there are over 700 


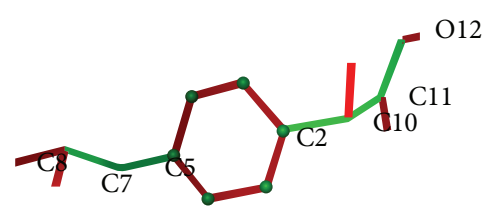

FIGURE 1: Rotatable bonds of ibuprofen (green bonds as rotatable bonds).

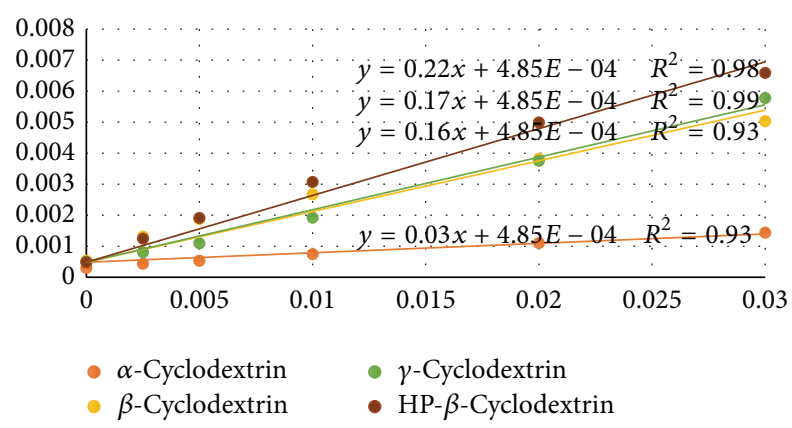

Figure 2: Phase-solubility diagrams of ibuprofen in aqueous CD solutions at $25^{\circ} \mathrm{C}$.

research papers about theoretical calculations on cyclodextrins complexation with various drugs until 2014. These theoretical calculations could be classified into four different types, such as quantum mechanics (QM) [7], molecular dynamics (MD) [8], docking [9], and quantitative structure activity relationships (QSARs) [10]. However, the results from different modeling and experimental approaches were conflicting and contradictory. For example, ibuprofen and cyclodextrin complexes have been studied using different experimental techniques [11, 12]. There are also a few theoretical research papers on the complexation of ibuprofen and cyclodextrins $[13,14]$. However, these experimental and theoretical studies obtained various results. Some recent research found the binding free energy between ibuprofen and $\beta$-CD was stronger than complex of ibuprofen and HP$\beta$-CD [11, 12]. However, Mura (1998) found the HP- $\beta$-CD solubilizing effectiveness for ibuprofen was stronger than $\beta$-CD in aqueous environment [12]. Núñez-Agüero (2006) used MM-GBSA method to calculate the enthalpy between ibuprofen and $\beta-C D$ [15]. The results from recent researches were summarized in Table 1.

Thus, it is necessary to establish a standard method to investigate the complexation between cyclodextrin and drug. The purpose of this study was to compare different simulation methods of ibuprofen and cyclodextrin complexation. Ibuprofen was chosen as a model drug.

\section{Simulation Methods}

2.1. Model Building of Drug and Cyclodextrins. The crystal structures of alpha $(\alpha)$, beta $(\beta)$, gamma $(\gamma)$ cyclodextrins were taken from The Cambridge Crystallographic Data Centre (CCDC) ( $\alpha$-CD for "BAJJAX," $\beta$-CD for "ARUXIU," and $\gamma$-CD for "CYDXPL"). Hydroxypropyl-beta-CD (HP- $\beta$-CD) was modified from the structure of $\beta$-CD using Discovery Studio Visualizer. Ibuprofen was built by Discovery Studio Visualizer. The structure of all molecules was optimized with a fast, Dreiding-like force field by Discovery Studio Visualizer.

2.2. Docking Simulation Setting. AutoDock Vina was one of widely used docking programs, which can predict free energy of ligand-receptor interaction [20]. In our research, the AutoDock Tools package (version 1.5.6) and AutoDock Vina (version 1.1.2) were used to perform docking studies [9].

In docking simulation, ibuprofen was used as a ligand, while cyclodextrins were used as receptor. Before the docking, AutoDock Tools was used to optimize the structure of ibuprofen from the PDB files. Atom C2 of ibuprofen was chosen to be the root atom because all its bonds to other atoms were rotatable. AutoDock Tools was used to determine the possible rotations of bonds. Current total five rotatable bonds were the bonds between atom $\mathrm{C} 8$ and $\mathrm{C}$, C7 and C5, C2 and C10, C10 and C11, and C11 and O12 as shown in Figure 1. The pdbqt format files (required as input to AutoDock Vina) were generated using AutoDock Tools. During the docking procedure, ibuprofen was docked to cyclodextrin molecules using the following Cartesian coordinates as the center of the search space: $x=2.5 \AA$, $y=6.5 \AA$, and $z=7.5 \AA$. The dimensions of docking grid were $60 \AA \times 60 \AA \times 60 \AA$. All the other parameters were the default values by AutoDock Vina. The docking pose with the highest score was treated as the stable binding model. The docking score from AutoDock Vina output was used as the binding free energy.

2.3. MD Simulation. The stable binding model from docking was used as the starting structure of MD simulation. The MD simulation was performed by the Amber14 and Amber Tools 14 software package. Currently, there is no specific force field for cyclodextrin molecules. Unlike the traditional Amber force fields, general Amber force field (GAFF) is more general for organic chemical [21]. Some recent articles chose GAFF force fields in MD simulation of CDs [22-24]. So the GAFF force field was selected in Antechamber module with AM1BCC charge method for all molecules. A water cube of $10 \AA$ thickness was added to solvate the system with the TIP3P water model. All MD setting details were shown in Table 2. After two stages of minimization, $30 \mathrm{~ns} \mathrm{MD}$ simulations were performed. The detailed simulation protocol was similar to our previous simulation researches [25-27].

2.4. QM/MM Simulation. The stable binding model from docking was used as the starting structure of QM simulation. Coupled potential QM/MM simulation has been performed in Amber Tools 14. The complex was masked as quantum mechanics (QM) modeling by the semiempirical PM3 Hamiltonian method, while all water molecules were treated as classical MM. The periodic boundaries and PME approaches were used to calculate the long-range QM-MM electrostatic energies and forces and the long-range QM-QM forces with $8 \AA$ cutoff distance in both two stages. The SHAKE algorithm 


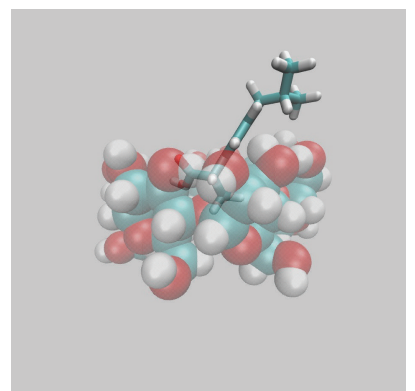

(1)

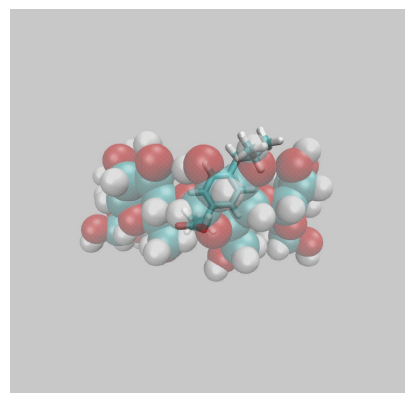

(1)

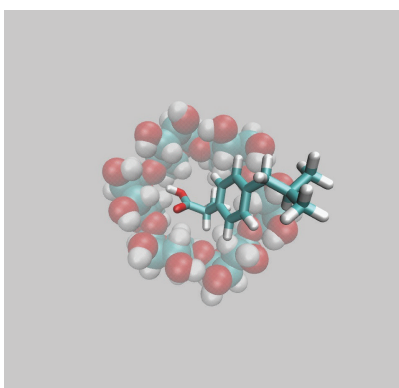

(2)

(a)

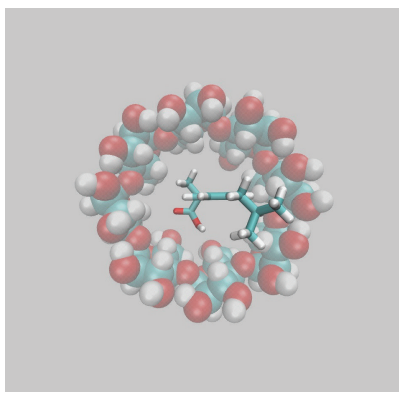

(2)

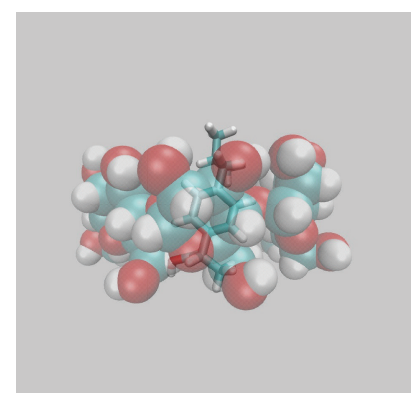

(1)

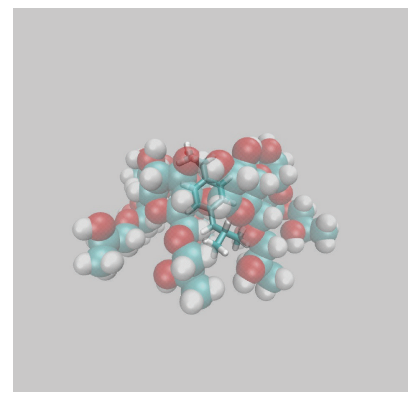

(1)

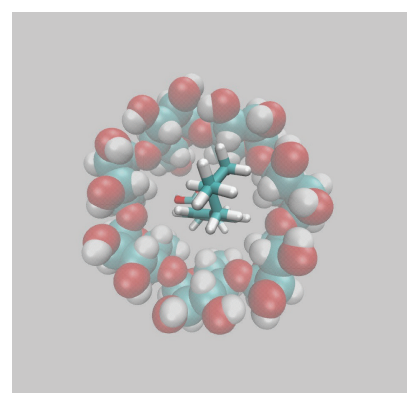

(2)

(b)

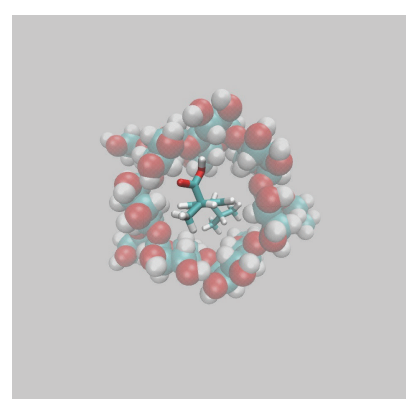

(2)

(d)

Figure 3: Optimal models of the inclusion complexation by AutoDock Vina. (a) $\alpha$-CD and IBF; (b) $\beta$-CD and IBF; (c) $\gamma$-CD and IBF; (d) HP- $\beta$-CD and IBF. ((1) was the side view and (2) was top view from the wide rim of cyclodextrin; Cyclodextrin molecules were shown as semitransparent for clarity.)

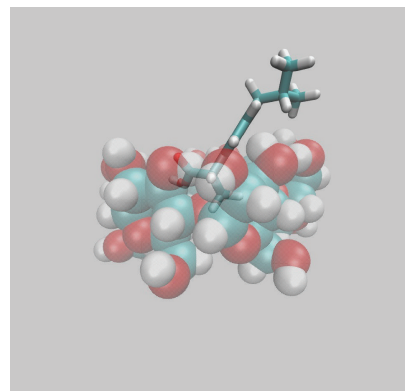

(a)

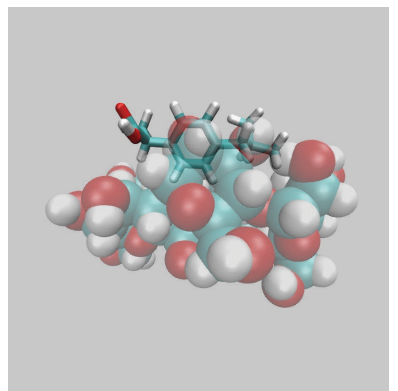

(b)

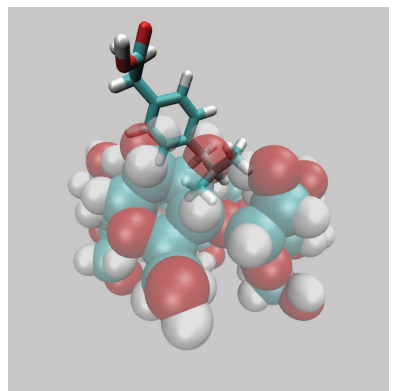

(c)

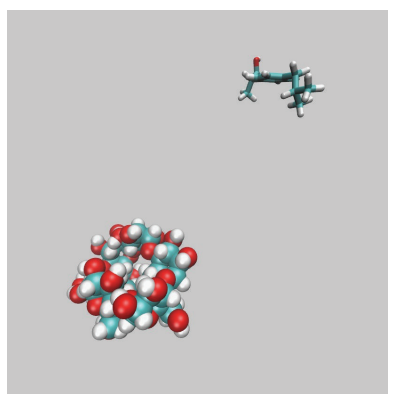

(d)

FIGURE 4: Snapshots of $\alpha$-CD and IBF complexation by $30 \mathrm{~ns}$ MD simulation. (a) 0 ns; (b) 9 ns; (c) $15 \mathrm{~ns}$; (d) 24 ns. (Cyclodextrin molecules were shown as semitransparent for clarity.)
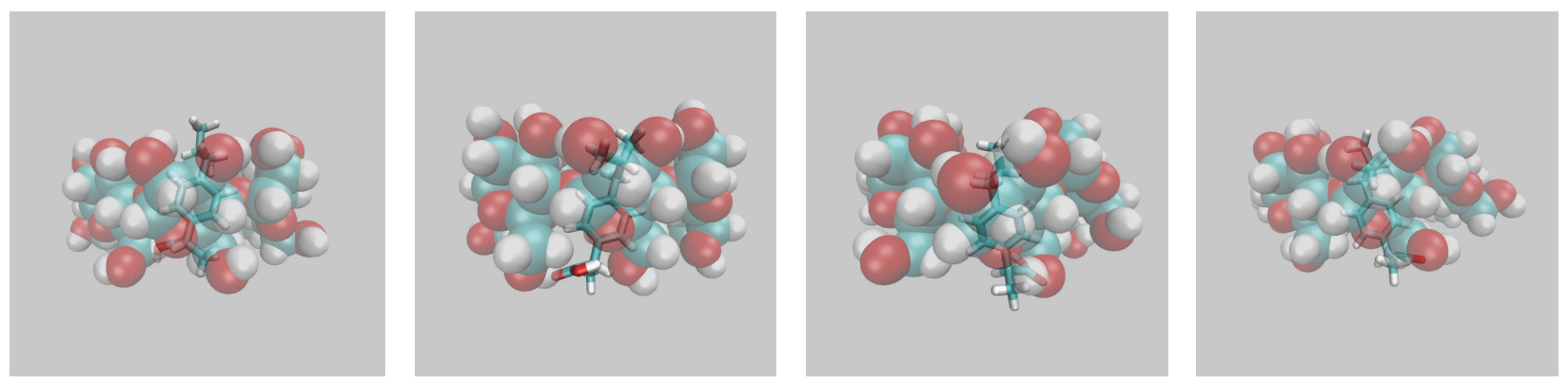

Figure 5: Structures of $\beta$-CD and IBF complexes obtained by the MD method every $10 \mathrm{~ns}$. 

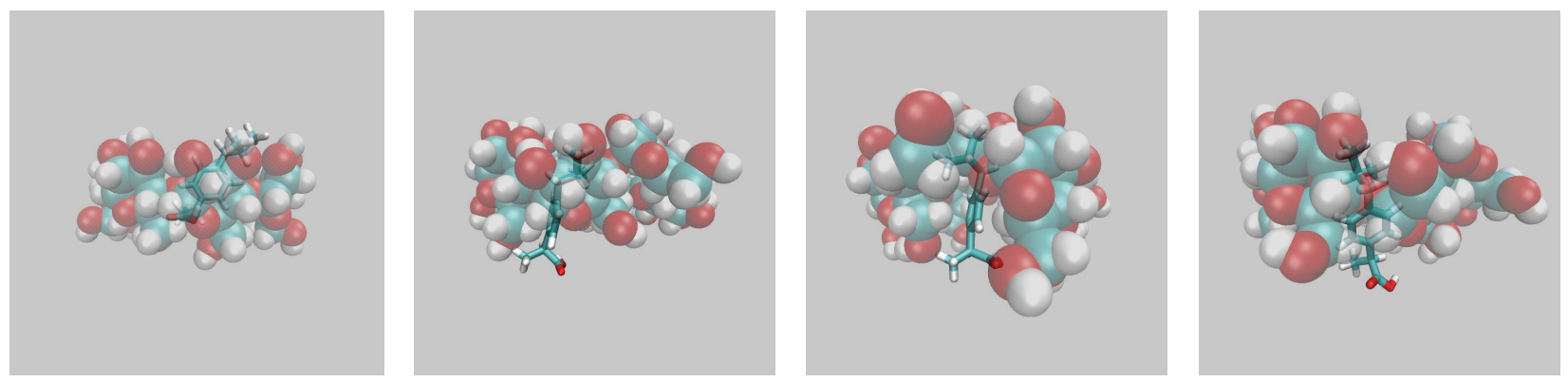

FIGURE 6: Structures of $\gamma$-CD and IBF complexes obtained by the MD method every $10 \mathrm{~ns}$.
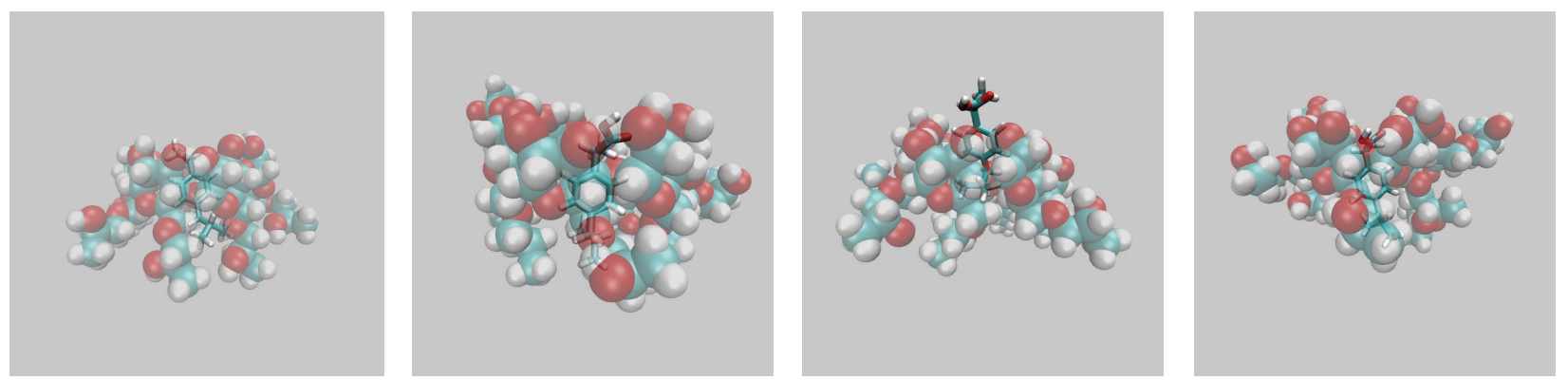

Figure 7: Structures of HP- $\beta$-CD and IBF complexes obtained by the MD method every $10 \mathrm{~ns}$. (Cyclodextrin molecules were shown as semitransparent for clarity.)

was applied to QM atoms in order to constrain all bonds involving hydrogen.

In case of ibuprofen and cyclodextrin simulation in water, the system was optimized with the 200 -steps steepest descent method and then 300-steps conjugate gradient method. Then the system was heated up to $300 \mathrm{~K}$. In QM/MM stage, the system carried out $1 \mathrm{~ns}$ simulation at $300 \mathrm{~K}$ with an average pressure of $1 \mathrm{~atm}$ by using Langevin dynamics with the collision frequency $1 \mathrm{ps}^{-1}$.

\subsection{MM-PBSA Method for Binding Free Energy Calculation.} MM-PBSA method was chosen to calculate the binding free energy [28]. In this study, the binding free energy of the ibuprofen and CD inclusion complex $\left(\Delta G_{\text {bind }}\right)$ was calculated by the free energy of complex $\left(\Delta G_{\text {complex }}\right)$ and the isolated ibuprofen $\left(\Delta G_{\text {ibuprofen }}\right)$ and $\operatorname{CDs}\left(\Delta G_{\mathrm{CD}}\right)$ as the following equation:

$$
\Delta G_{\text {bind }}=\Delta G_{\text {complex }}-\Delta G_{\text {ibuprofen }}-\Delta G_{\mathrm{CD}} .
$$

The Gibbs free energy $(\Delta G)$ was calculated by the enthalpy $(\Delta H)$ and entropy with invariable temperature $(T \Delta S)$ :

$$
\Delta G=\Delta H-T \Delta S .
$$

2.6. Phase-Solubility Diagram. Over amount of ibuprofen was added in the $10 \mathrm{~mL}$ of cyclodextrin solution with the concentration of $0,0.0025 \mathrm{M}, 0.005 \mathrm{M}, 0.01 \mathrm{M}, 0.02 \mathrm{M}$, and $0.03 \mathrm{M}$, respectively. The solutions were sonicated for 60 minutes to reach the equilibration. After the samples were filtered, the filtrate was measured by UV-visual Spectrometer at the wavelength of $264 \mathrm{~nm}$. The experimental result was shown in Figure 2.

The stability constants of ibuprofen and CDs complexes in water were calculated by the following equation:

$$
K=\frac{\text { slope }}{\text { intercept }(1-\text { slope })} \text {. }
$$

\section{Result and Discussion}

3.1. Docking Result. The molecular docking is an attractive strategy for ligand-receptor interaction. In our study docking was conducted to calculate the binding energies between ibuprofen and cyclodextrins complexes by AutoDock Vina. Figure 3 showed the optimal models for ibuprofen and CDs. Unlike the interaction of $\alpha-\mathrm{CD} /$ ibuprofen complex, the carboxylic part of ibuprofen inserted deeply into the cavity of $\beta$ - and $\gamma$-CD molecules due to their large cavity. Moreover, ibuprofen molecule had a slight inclination in the cavity of $\gamma$-CD because $\gamma$-CD has the largest cavity.

According to the docking results, the binding energies between ibuprofen and cyclodextrins were $-3.6,-4.4$, -4.2 , and $-5.0 \mathrm{kcal} / \mathrm{mol}$, respectively. The binding energy of ibuprofen and $\alpha$-CD was the lowest value among four complexes, which confirmed the shallow structure among them. Ibuprofen and HP- $\beta$-CD complex had the strongest binding energy. The possible reason is that the interactions between hydroxypropyl group of HP- $\beta-\mathrm{CD}$ and isopropyl group of ibuprofen make the complex more stable. The negative binding affinity energy values suggested that the 

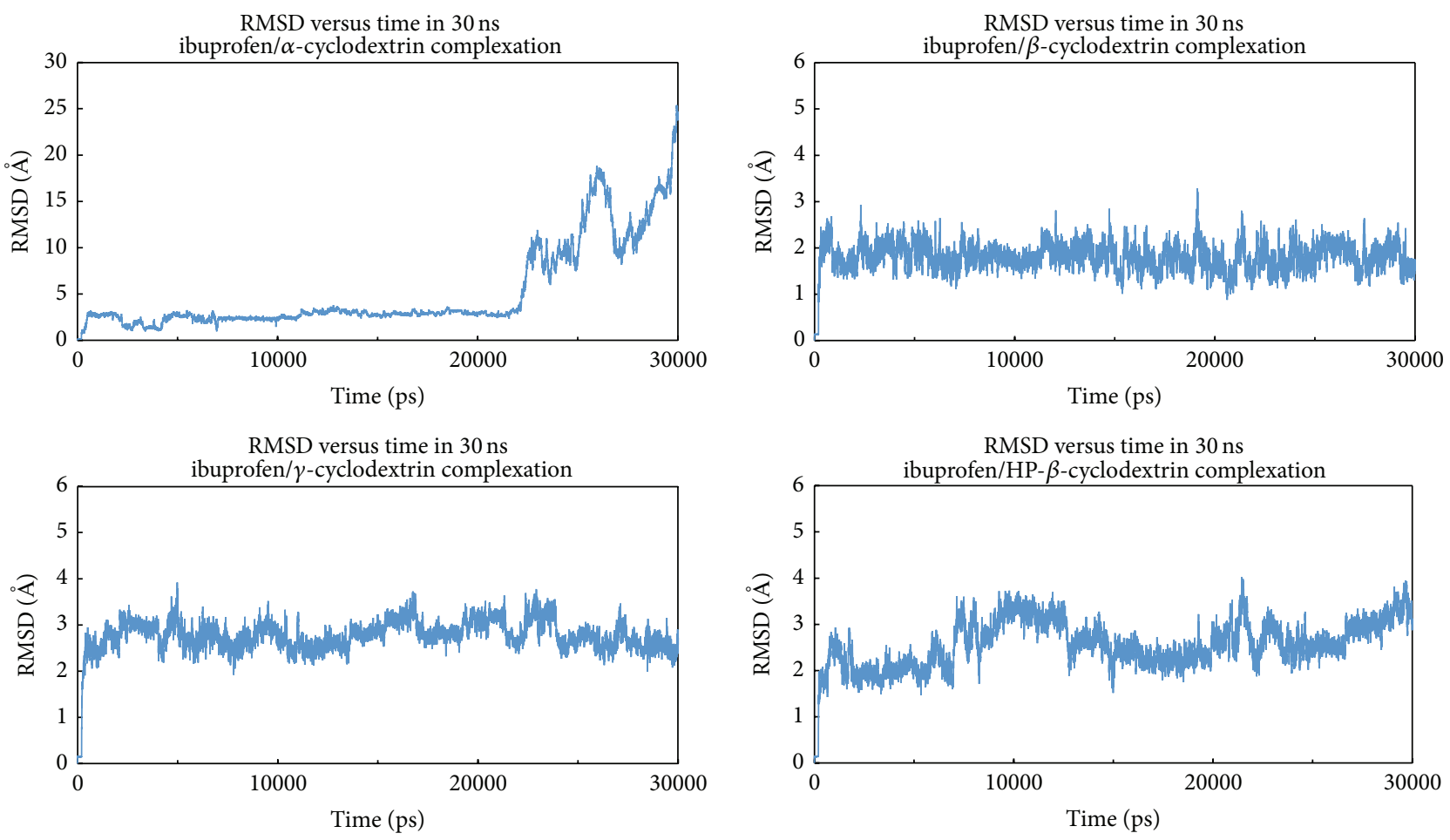

FIGURE 8: The RMSD plots of backbone atoms for the simulations of four complexes.

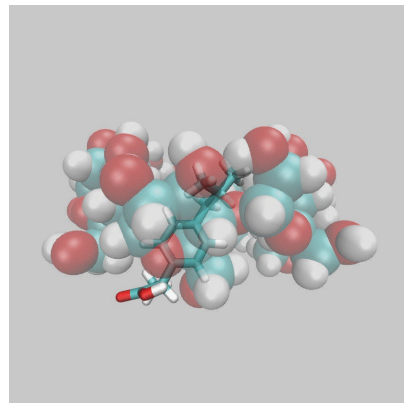

(a)

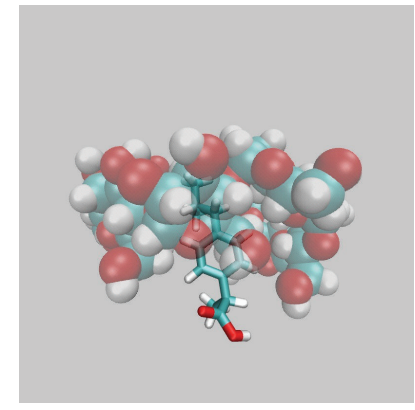

(b)

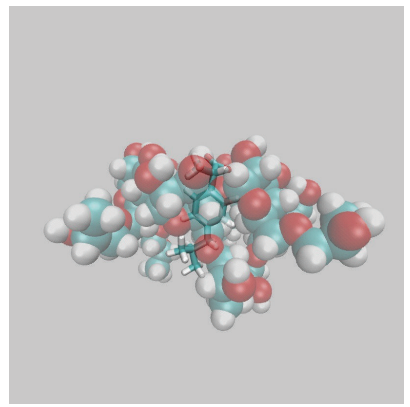

(c)

FIGURE 9: Structures of CDs and IBF complexes obtained by the QM/MM method. (Cyclodextrin molecules were shown as semitransparent for clarity.) (a) IBF and $\beta$-CD; (b) IBF and $\gamma$-CD; (c) IBF and HP- $\beta$-CD.

formation of all these inclusion complexes was a spontaneous reaction.

3.2. MD Trajectory Analysis. Figure 4 showed the snapshots during the $30 \mathrm{~ns} \mathrm{MD}$ simulations of ibuprofen and $\alpha$-CD. These snapshots indicated the unstable state of the complex. At the first $6 \mathrm{~ns}$, the structure of ibuprofen and $\alpha$-CD complex did not change much. After that, the ibuprofen started to reverse its structure. After 24 ns, the ibuprofen separated from $\alpha$-CD. However, the complex of ibuprofen and $\alpha$-CD was found to be unstable during another three MD simulations. The possible reason may be the weak binding energy of ibuprofen $/ \alpha$-CD complex, which is also confirmed by the low solubilisation of $\alpha$-CD to ibuprofen in the experiments.
Figures 5-7 showed the stable structures between ibuprofen and other three cyclodextrin molecules during the $30 \mathrm{~ns}$ simulation. In the simulation, the isopropyl group of ibuprofen totally inserted into the cavity of $\beta$-CD and the carboxylic part will stick out from the primary side of $\beta$-CD, which was in agreement with previous study about crystal structure of ibuprofen/ $\beta$-CD complex [29]. However, our result was different from previous simulation papers [15]. The possible reason was improper force field and too short simulation time ( $4.5 \mathrm{~ns})$ in previous simulations. For $\gamma$-CD, ibuprofen lied in the cavity with about $45^{\circ}$ inclination angle. In addition, some portion of the $\gamma$-CD molecule became twisted to fit the inclusion ibuprofen. This phenomenon was not found in docking studies because all CD molecules were treated as the rigid structure in the docking. Figure 7 displayed that 
TABLE 1: Recent researches about ibuprofen and CDs complexes.

\begin{tabular}{|c|c|c|c|}
\hline $\mathrm{CD}$ & Binding energy $(\mathrm{kcal} / \mathrm{mol})$ & Method & Reference \\
\hline$\beta-\mathrm{CD}$ & -16.90 & MM/GBSA & [15] \\
\hline$\beta-\mathrm{CD}$ & -6.31 & Phase-solubility analysis & [13] \\
\hline$\beta-\mathrm{CD}$ & -5.45 & \multirow{2}{*}{ Phase-solubility analysis } & \multirow{2}{*}[12]{} \\
\hline $\mathrm{HP}-\beta-\mathrm{CD}$ & -5.09 & & \\
\hline $\mathrm{HP}-\beta-\mathrm{CD}\left(\mathrm{MS}^{\mathrm{a}}=0.29\right)$ & -3.66 & Phase-solubility analysis & [16] \\
\hline$\alpha-\mathrm{CD}$ & -3.83 & \multirow{3}{*}{ Titration calorimetry } & \multirow{3}{*}[14]{} \\
\hline$\beta-\mathrm{CD}$ & -5.59 & & \\
\hline$\gamma$-CD & -2.92 & & \\
\hline$\beta-\mathrm{CD}$ & -5.24 & \multirow{3}{*}{ Titration calorimetry } & \multirow{3}{*}[11]{} \\
\hline $\mathrm{HP}-\beta-\mathrm{CD}(\mathrm{MS}=0.62)$ & -4.55 & & \\
\hline $\mathrm{HP}-\beta-\mathrm{CD}(\mathrm{MS}=0.87)$ & -5.05 & & \\
\hline HP- $\beta$-CD (in citric buffer) & -4.92 & \multirow{2}{*}{ Titration calorimetry } & \multirow{2}{*}[17]{} \\
\hline HP- $\beta$-CD (in phosphate buffer) & -4.80 & & \\
\hline$\beta$-CD (protonated) & -5.36 & \multirow{2}{*}{ Fluorescence spectrometry } & \multirow{2}{*}[18]{} \\
\hline$\beta$-CD (deprotonated) & -5.79 & & \\
\hline$\beta-\mathrm{CD}$ & -5.41 & Circular dichroism spectropolarimetry & [19] \\
\hline
\end{tabular}

${ }^{a}$ MS: molar degrees of substitution.

TABLE 2: Simulated cell compositions and atom numbers for ibuprofen and cyclodextrin complexes.

\begin{tabular}{|c|c|c|c|c|}
\hline & $\mathrm{IBF} / \alpha-\mathrm{CD}$ & $\mathrm{IBF} / \beta-\mathrm{CD}$ & $\mathrm{IBF} / \gamma-\mathrm{CD}$ & $\mathrm{IBF} / \mathrm{HP}-\beta-\mathrm{CD}$ \\
\hline Water shell $(\AA)$ & 10 & 10 & 10 & 10 \\
\hline Atom number of ibuprofen & 33 & 33 & 33 & 33 \\
\hline Atom number of $\mathrm{CD}$ & 126 & 147 & 168 & 217 \\
\hline Molecule number of water & 1213 & 1341 & 1489 & 1793 \\
\hline Number of counterions & 0 & 0 & 0 & 0 \\
\hline Total atom number & 3798 & 4203 & 4668 & 5899 \\
\hline
\end{tabular}

TABLE 3: MM-PBSA binding free energy $(\mathrm{kcal} / \mathrm{mol})$ and energy components.

\begin{tabular}{lccc}
\hline & $\beta$-CD \& IBF & $\gamma$-CD \& IBF & HP- $\beta$-CD \& IBF \\
\hline$\Delta E_{\mathrm{ELE}}$ & $-2.03 \pm 1.40$ & $-2.68 \pm 1.71$ & $-10.01 \pm 2.33$ \\
$\Delta E_{\mathrm{VDW}}$ & $-22.83 \pm 1.30$ & $-22.92 \pm 1.46$ & $-28.06 \pm 1.94$ \\
$\Delta E_{\mathrm{INT}}$ & $-0.00 \pm 0.01$ & $-0.00 \pm 0.01$ & $0.00 \pm 0.01$ \\
$\Delta E_{\mathrm{GAS}}$ & $-24.86 \pm 2.09$ & $-25.60 \pm 2.32$ & $-38.07 \pm 2.30$ \\
$\Delta E_{\mathrm{PBSUR}}$ & $-2.49 \pm 0.07$ & $-2.58 \pm 0.07$ & $-2.78 \pm 0.10$ \\
$\Delta E_{\mathrm{PBCAL}}$ & $12.09 \pm 2.06$ & $14.31 \pm 2.67$ & $21.84 \pm 1.94$ \\
$\Delta E_{\mathrm{PBSOL}}$ & $9.60 \pm 2.03$ & $11.73 \pm 2.64$ & $19.06 \pm 1.97$ \\
$\Delta E_{\mathrm{PBTOT}}$ & $-15.26 \pm 1.34$ & $-13.87 \pm 2.17$ & $-19.00 \pm 2.46$ \\
\hline$T \Delta S_{\mathrm{TRA}}$ & $-12.41 \pm 0.00$ & $-12.42 \pm 0.00$ & $-12.44 \pm 0.00$ \\
$T \Delta S_{\mathrm{ROT}}$ & $-9.68 \pm 0.01$ & $-9.70 \pm 0.01$ & $-9.74 \pm 0.01$ \\
$T \Delta S_{\mathrm{VIB}}$ & $10.49 \pm 2.83$ & $8.93 \pm 3.50$ & $7.05 \pm 3.08$ \\
$T \Delta S_{\mathrm{TOT}}$ & $-11.59 \pm 2.83$ & $-13.20 \pm 3.50$ & $-15.13 \pm 3.08$ \\
\hline$\Delta G_{\mathrm{PB}}(\Delta E-T \Delta S)$ & -3.67 & -0.67 & -3.87 \\
\hline
\end{tabular}

ibuprofen binding with $\mathrm{HP}-\beta-\mathrm{CD}$ on the opposite direction to $\beta$-and $\gamma$-CD. The possible reason was nonpolar interaction between hydroxypropyl group of HP- $\beta$-CD and isopropyl part of ibuprofen. The MD simulation predicted model was in agreement with previous research about the spectroscopic
TABLE 4: Stability constants of ibuprofen with CDs.

\begin{tabular}{lcccc}
\hline $\begin{array}{l}\Delta G \\
(\mathrm{kcal} / \mathrm{mol})\end{array}$ & Experiments & Docking & $\mathrm{MD}$ & $\begin{array}{c}\text { QM/MM } \\
\text { (enthalpy only) }\end{array}$ \\
\hline$\alpha$-CD & -2.48 & -3.6 & $\mathrm{n} / \mathrm{a}$ & $\mathrm{n} / \mathrm{a}$ \\
$\beta$-CD & -3.58 & -4.4 & $-3.67 \pm 3.13$ & $-17.22 \pm 1.40$ \\
$\gamma$-CD & -3.60 & -4.2 & $-0.67 \pm 4.12$ & $-14.75 \pm 1.33$ \\
$\mathrm{HP}-\beta$-CD & -3.78 & -5.0 & $-3.87 \pm 3.94$ & $-20.28 \pm 1.56$ \\
\hline
\end{tabular}

characterization of ibuprofen and 2-hydroxypropyl- $\beta$-CD inclusion complex [16].

Figure 8 showed the root mean square displacement (RMSD) for backbone heavy atoms of the complexes in $30 \mathrm{~ns}$ MD simulation. RMSD value of $\alpha$-CD/ibuprofen complex increased sharply after $20 \mathrm{~ns}$ MD simulation, which agreed with the structural change in Figure 4 . However, for the complexes ibuprofen and $\beta-, \gamma-$, and HP- $\beta-C D$, the RMSD values were relatively stable, which indicated the complexes had reached the equilibrated state after $30 \mathrm{~ns}$ simulation. Therefore, only the inclusion complex of $\beta-, \gamma^{-}$, and HP- $\beta$ $\mathrm{CD}$ was further calculated for binding free energy and QM modeling. 
The MM-PBSA approach is a useful tool to calculate the binding free energies. The $100 \mathrm{MD}$ snapshots from the last $1 \mathrm{~ns}$ of each system were used for binding free energy calculations. The binding free energies $\left(\Delta G_{\mathrm{PB}}\right)$ and the other energy contributions were shown in Table 3. The van der Waals force $\left(\Delta E_{\mathrm{VDW}}\right)$ made the key contribution to the complexation between ibuprofen and cyclodextrin molecules. The $\Delta E_{\mathrm{VDW}}$ contribution of the HP- $\beta$-CD complex was stronger than those of $\beta$ - and $\gamma$-CD complexes. The results of $\Delta E_{\text {Рвтот }}$ were not accurately equal to the binding free energy because the entropy contribution was not estimated. Thus, $\Delta G_{\mathrm{PB}}$ $\left(\Delta E_{\mathrm{PBTOT}}-T \Delta S_{\mathrm{TOT}}\right)$ were calculated for the Gibbs free energy of the complexes. The binding energies of $\beta$ - and HP$\beta$-CD complex are -3.67 and $-3.87 \mathrm{kcal} / \mathrm{mol}$, respectively, which were close to the experimental values $(-3.58$ and $-3.78 \mathrm{kcal} / \mathrm{mol}$ ) in Table 4 . These results suggested that the entropy and solvation contribution were paramount for the binding free energy between ibuprofen and cyclodextrin molecules. MD simulation had very good prediction for ibuprofen with $\beta$-CD and HP- $\beta$-CD complex, but not for complex of $\gamma$-CD. The possible reason may be the binding mode of $\gamma$-CD and ibuprofen was not 1:1 because of the large cavity of $\gamma$-CD.

3.3. $Q M / M M$ Result. Due to the separation of $\alpha-C D$ and ibuprofen in MD simulation, we only calculated the QM/MM simulation for ibuprofen with $\beta$-, $\gamma$-, and HP- $\beta$ $\mathrm{CD}$ complexes. In QM/MM simulation, the binding modes of ibuprofen and CDs were shown in Figure 9, which were very similar to the models in MD simulation. The PM3 theory was used for quantum calculation. The QM/MM-GBSA method predicted the enthalpy of the ibuprofen with $\beta-, \gamma$-, and HP$\beta$-CD complexes being comparatively close to the enthalpy values in MD simulation, as shown in Tables 3 and 4 . However, QM calculation took much longer computing time than MD simulations and the obtained energy magnitudes were far off from the experimental values because the entropy was not included. Thus, QM simulation is not recommended for in silico formulation screening.

In this paper, three different kinds of computer modeling methods were used to investigate the complexation of ibuprofen with $\alpha-, \beta-, \gamma$-, and HP- $\beta$-CD. The binding energies of AutoDock Vina results show stronger binding of HP- $\beta$-CD than other complexes. However, the values from docking were a little overestimated compared to experimental data. MM-PBSA approaches estimated that the VDW mainly contributed to the complexation formation. In addition, the entropy and solvation contribution were very important for the binding energy calculation, which was ignored in previous researches [15]. The binding free energy from MD simulation was very close to experimental results. QM/MM simulation predicted the similar enthalpy of ibuprofen with $\beta$-, $\gamma$-, and HP- $\beta$-CD with MD simulation, but QM simulation took significantly longer simulation time. In comparison to the experimental value, the values from docking results for all complexes were found a little overestimated, but the ranking between them were correct. MD simulation had very good prediction for ibuprofen with $\beta-\mathrm{CD}$ and $\mathrm{HP}-\beta-\mathrm{CD}$ complex, but not for complex of $\gamma$-CD.

\section{Conclusion}

In this study, three modeling methods were used to investigate the complexation of ibuprofen and CDs in water. All modeling and experimental results showed that HP- $\beta-C D$ inclusion was stronger than other complexes. Docking result has similar ranking with experiments. The Gibbs free energy for $\beta$-CD and HP- $\beta$-CD complex from MD simulations and MM-PBSA method were the most close to the experimental value. QM method took too expensive computing resource to use in the wet laboratory. MD simulation with MMPBSA method may be fit to in silico screen for cyclodextrin formulations.

\section{Conflict of Interests}

The authors declare that there is no conflict of interests regarding the publication of this paper.

\section{Acknowledgments}

This study was supported by Science and Technology Development Fund (FDCT), Macao SAR, (074-2012-A3) and Research Fund of University of Macau (MRG013-WYT-2013ICMS and CPG2014-00012-ICMS).

\section{References}

[1] T. Loftsson and M. E. Brewster, "Pharmaceutical applications of cyclodextrins: basic science and product development," Journal of Pharmacy and Pharmacology, vol. 62, no. 11, pp. 1607-1621, 2010.

[2] T. Loftsson and M. E. Brewster, "Pharmaceutical applications of cyclodextrins: effects on drug permeation through biological membranes," Journal of Pharmacy and Pharmacology, vol. 63, no. 9, pp. 1119-1135, 2011.

[3] R. Singh, N. Bharti, J. Madan, and S. N. Hiremath, "Characterization of cyclodextrin inclusion complexes-a review," Journal of Pharmaceutical Science and Technology, vol. 2, no. 3, pp. 171$183,2010$.

[4] G. S. Jadhav and P. R. Vavia, "Physicochemical, in silico and in vivo evaluation of a danazol- $\beta$-cyclodextrin complex," International Journal of Pharmaceutics, vol. 352, no. 1-2, pp. 5$16,2008$.

[5] M. Bonini, S. Rossi, G. Karlsson, M. Almgren, P. L. Nostro, and P. Baglioni, "Self-assembly of $\beta$-cyclodextrin in water. Part 1 : Cryo-TEM and dynamic and static light scattering," Langmuir, vol. 22, no. 4, pp. 1478-1484, 2006.

[6] M. Dab̧rowska, J. Krzek, and E. Miękina, "Stability analysis of cefaclor and its inclusion complexes of $\beta$-cyclodextrin by thin-layer chromatography and densitometry," Journal of Planar Chromatography-Modern TLC, vol. 25, no. 2, pp. 127-132, 2012.

[7] R. Djemil and D. Khatmi, "Quantum mechanical study of complexation of dopamine and epinephrine with $\beta$-Cyclodextrin using PM6, ONIOM and NBO analysis," Journal of Computational and Theoretical Nanoscience, vol. 9, no. 10, pp. 1571-1576, 2012. 
[8] W. Wang, O. Donini, C. M. Reyes, and P. A. Kollman, "Biomolecular simulations: recent developments in force fields, simulations of enzyme catalysis, protein-ligand, proteinprotein, and protein-nucleic acid noncovalent interactions," Annual Review of Biophysics and Biomolecular Structure, vol. 30, no. 1, pp. 211-243, 2001.

[9] O. Trott and A. J. Olson, "AutoDock Vina: improving the speed and accuracy of docking with a new scoring function, efficient optimization, and multithreading," Journal of Computational Chemistry, vol. 31, no. 2, pp. 455-461, 2010.

[10] M. A. Filippa, M. I. Sancho, and E. I. Gasull, "Determination of apparent binding constants by NSAIDs- $\beta$ cyclodextrin complexes: HPLC, phase solubility diagrams and theoretical studies," Journal of Inclusion Phenomena and Macrocyclic Chemistry, vol. 77, no. 1-4, pp. 223-230, 2013.

[11] M. di Cagno, P. C. Stein, N. Skalko-Basnet, M. Brandl, and A. Bauer-Brandl, "Solubilization of ibuprofen with $\beta$-cyclodextrin derivatives: energetic and structural studies," Journal of Pharmaceutical and Biomedical Analysis, vol. 55, no. 3, pp. 446-451, 2011.

[12] P. Mura, G. P. Bettinetti, A. Manderioli, M. T. Faucci, G. Bramanti, and M. Sorrenti, "Interactions of ketoprofen and ibuprofen with $\beta$-cyclodextrins in solution and in the solid state," International Journal of Pharmaceutics, vol. 166, no. 2, pp. 189-203, 1998.

[13] M. K. Ghorab and M. C. Adeyeye, "Elucidation of solution state complexation in wet-granulated oven-dried ibuprofen and $\beta$-cyclodextrin: FT-IR and 1H-NMR studies," Pharmaceutical Development and Technology, vol. 6, no. 3, pp. 315-324, 2001.

[14] S. Xing, Q. Zhang, C. Zhang, Q. Zhao, H. Ai, and D. Sun, "Isothermal titration calorimetry and theoretical studies on host-guest interaction of ibuprofen with $\alpha$-, $\beta$ - and $\gamma$ cyclodextrin," Journal of Solution Chemistry, vol. 38, no. 5, pp. 531-543, 2009.

[15] C.-J. Núñez-Agüero, C.-M. Escobar-Llanos, D. Díaz, C. Jaime, and R. Garduño-Juárez, "Chiral discrimination of ibuprofen isomers in $\beta$-cyclodextrin inclusion complexes: experimental (NMR) and theoretical (MD, MM/GBSA) studies," Tetrahedron, vol. 62, no. 17, pp. 4162-4172, 2006.

[16] I. Oh, M.-Y. Lee, Y.-B. Lee, S.-C. Shin, and I. Park, "Spectroscopic characterization of ibuprofen/2-hydroxypropyl- $\beta$ cyclodextrin inclusion complex," International Journal of Pharmaceutics, vol. 175, no. 2, pp. 215-223, 1998.

[17] G. L. Perlovich, M. Skar, and A. Bauer-Brandl, "Driving forces and the influence of the buffer composition on the complexation reaction between ibuprofen and HPCD," European Journal of Pharmaceutical Sciences, vol. 20, no. 2, pp. 197-200, 2003.

[18] J. L. Manzoori and M. Amjadi, "Spectrofluorimetric study of host-guest complexation of ibuprofen with $\beta$-cyclodextrin and its analytical application," Spectrochimica Acta, Part A: Molecular and Biomolecular Spectroscopy, vol. 59, no. 5, pp. 909916, 2003.

[19] J. C. Reijenga, B. A. Ingelse, and F. M. Everaerts, "Thermodynamics of chiral selectivity in capillary electrophoresis: separation of ibuprofen enantiomers with $\beta$-cyclodextrin," Journal of Chromatography A, vol. 792, no. 1-2, pp. 371-378, 1997.

[20] X. Zhang, S. E. Wong, and F. C. Lightstone, "Message passing interface and multithreading hybrid for parallel molecular docking of large databases on petascale high performance computing machines," Journal of Computational Chemistry, vol. 34, no. 11, pp. 915-927, 2013.
[21] J. Wang, R. M. Wolf, J. W. Caldwell, P. A. Kollman, and D. A. Case, "Development and testing of a general amber force field," Journal of Computational Chemistry, vol. 25, no. 9, pp. 1157-1174, 2004.

[22] S. Ö. Kocakaya, Y. Turgut, and N. Pirinççioglu, "Enantiomeric discrimination of chiral organic salts by chiral aza-15-crown5 ether with C 1 symmetry: experimental and theoretical approaches," Journal of Molecular Modeling, vol. 21, no. 3, pp. $1-13,2015$.

[23] S. J. Wallace, T. W. Kee, and D. M. Huang, "Molecular basis of binding and stability of curcumin in diamide-linked $\gamma$ cyclodextrin dimers," The Journal of Physical Chemistry B, vol. 117, no. 41, pp. 12375-12382, 2013.

[24] H. Zhao, Y. Zhu, M. Tong, J. He, C. Liu, and M. Tang, "Density functional theory studies on the inclusion complexes of cyclic decapeptide with 1-phenyl-1-propanol enantiomers," Journal of Molecular Modeling, vol. 18, no. 3, pp. 851-858, 2012.

[25] D. Ouyang, H. Zhang, H. S. Parekh, and S. C. Smith, "Structure and dynamics of multiple cationic vectors-siRNA complexation by all-atomic molecular dynamics simulations," The Journal of Physical Chemistry B, vol. 114, no. 28, pp. 9231-9237, 2010.

[26] D. Ouyang, H. Zhang, H. S. Parekh, and S. C. Smith, "The effect of $\mathrm{pH}$ on PAMAM dendrimer-siRNA complexationendosomal considerations as determined by molecular dynamics simulation," Biophysical Chemistry, vol. 158, no. 2-3, pp. 126133, 2011.

[27] D. Ouyang, H. Zhang, D.-P. Herten, H. S. Parekh, and S. C. Smith, "Structure, dynamics, and energetics of siRNA-cationic vector complexation: a molecular dynamics study," The Journal of Physical Chemistry B, vol. 114, no. 28, pp. 9220-9230, 2010.

[28] R. Luo, L. David, and M. K. Gilson, "Accelerated PoissonBoltzmann calculations for static and dynamic systems," Journal of Computational Chemistry, vol. 23, no. 13, pp. 1244-1253, 2002.

[29] S. S. Braga, I. S. Gonçalves, E. Herdtweck, and J. J. C. TeixeiraDias, "Solid state inclusion compound of S-ibuprofen in $\beta$ cyclodextrin: structure and characterisation," New Journal of Chemistry, vol. 27, no. 3, pp. 597-601, 2003. 

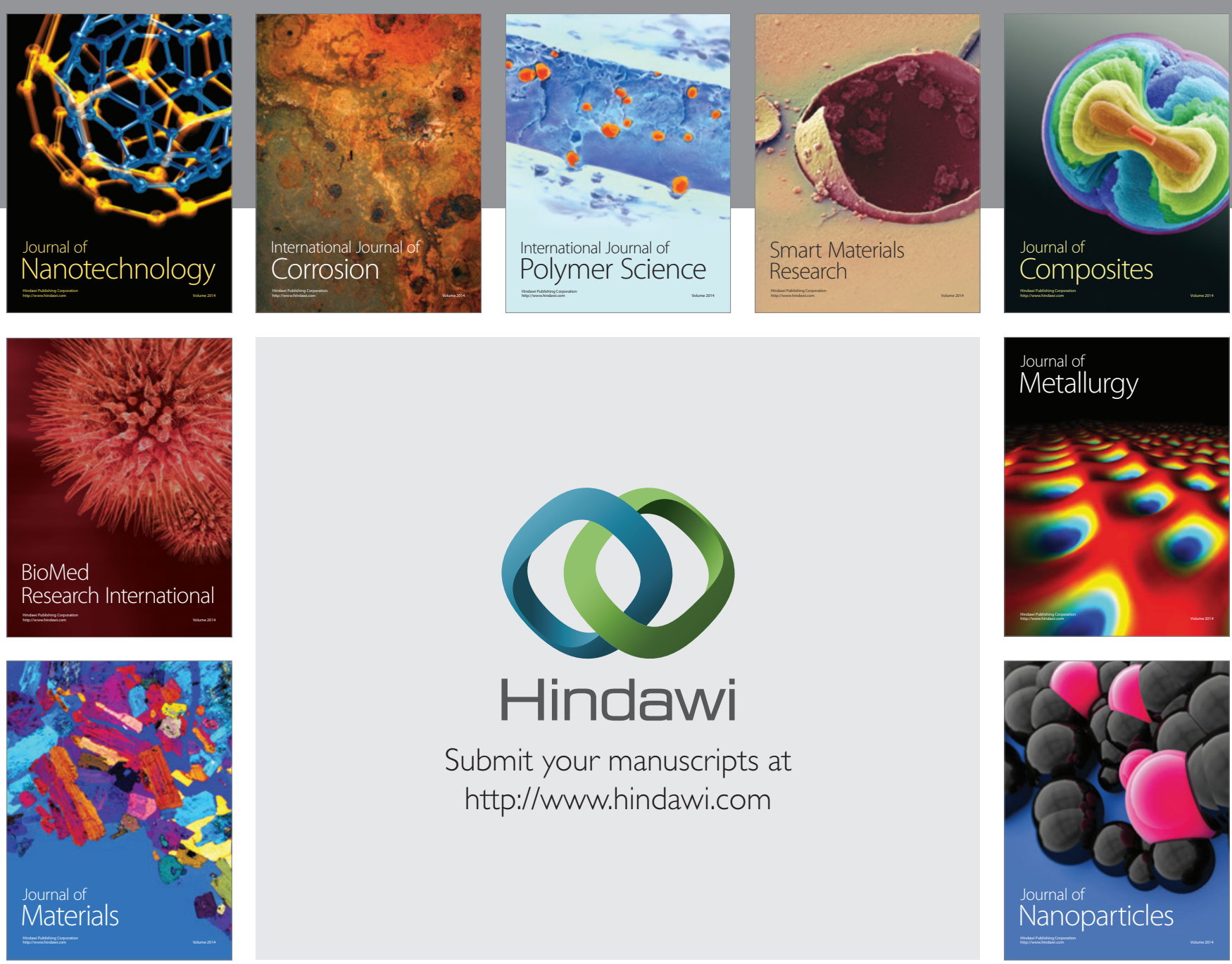

Submit your manuscripts at http://www.hindawi.com
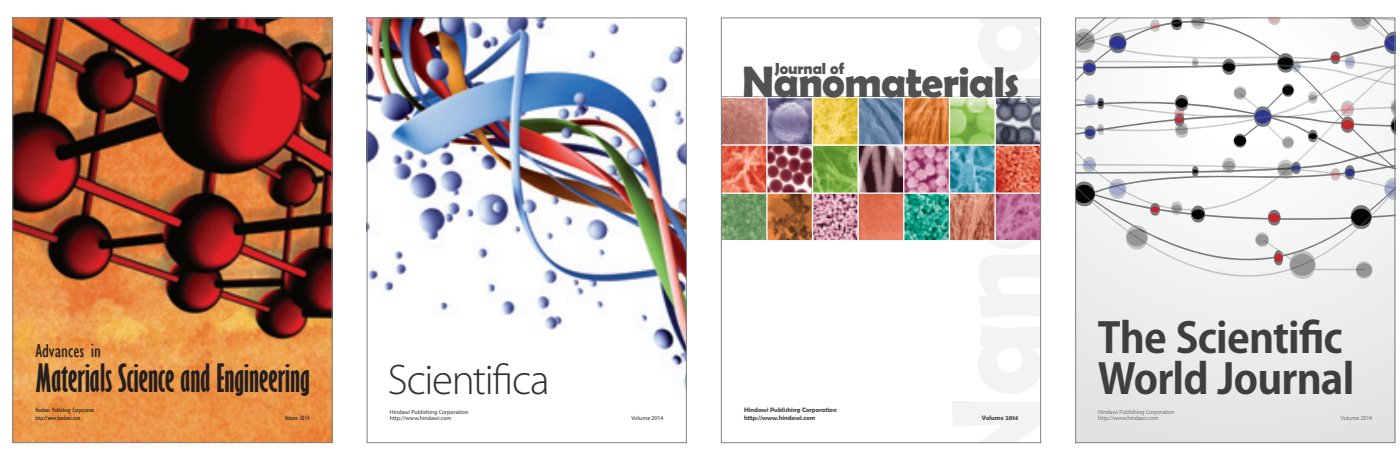

\section{The Scientific World Journal}
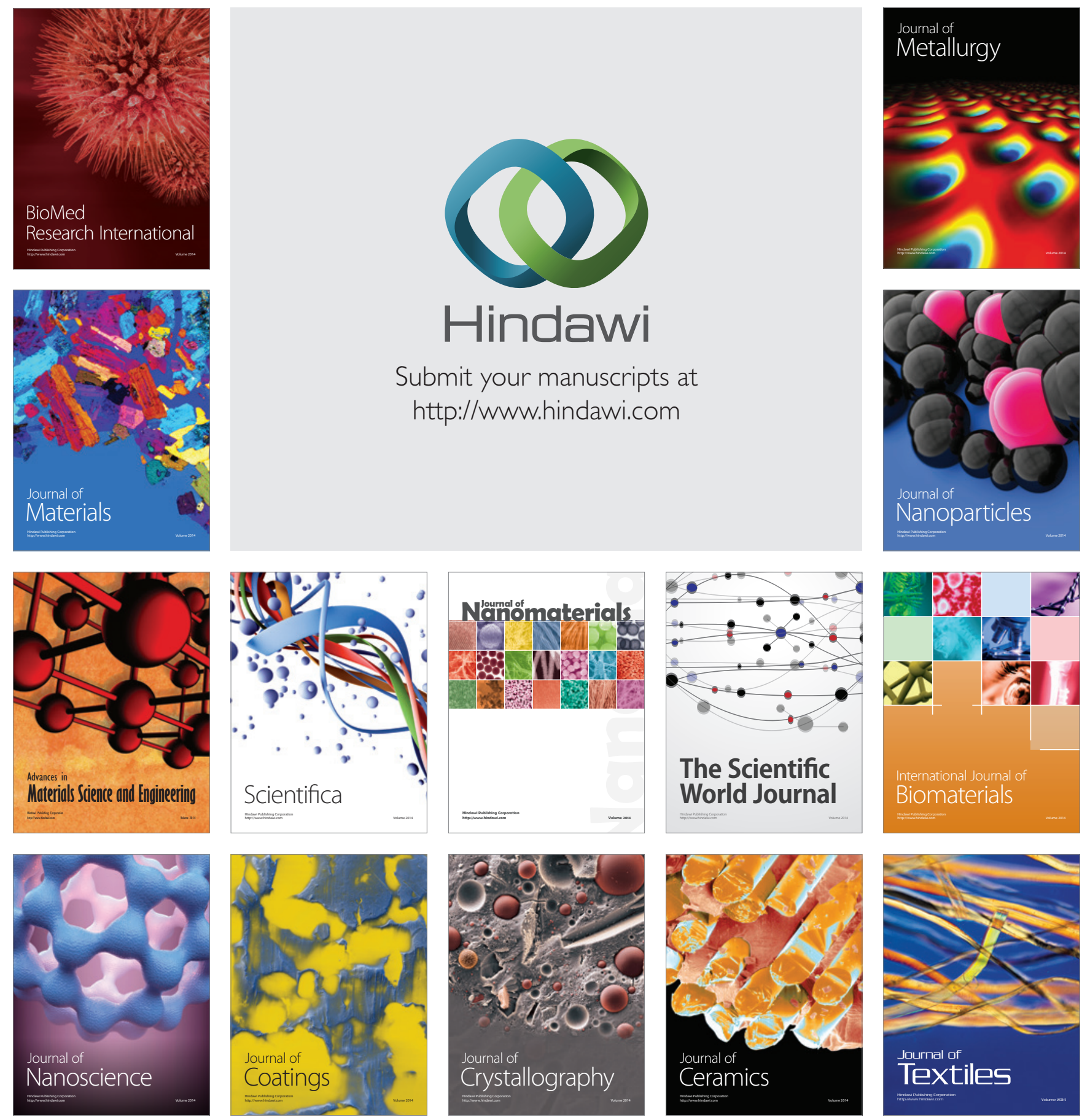